\title{
Fabrication of Chiral Poly(3,4-ethylenedioxythiophene) Derivatives Modified Glassy Carbon Electrodes for Ascorbic Acid Determination
}

\author{
Liqi Dong ${ }^{\dagger}$, Dufen $\mathrm{Hu}^{\dagger}$,Hui Sun, Xiaofei Zhu, Kaixin Zhang, Youshan, Zhang, Huakun Xing, Xuemin Duan* \\ $\uparrow$ These authors contributed equally to this work. \\ School of Pharmacy, \\ Jiangxi Science \& Technology Normal University, \\ Nanchang, 330013, P R China \\ e-mail:duanxuemin@126.com
}

\begin{abstract}
Two chiral poly(N-(tert-butoxycarbonyl)-Lphenylalayl (3,4-ethylenedioxythiophene-2'-yl)methylamide) (PEDOT-Boc-L-Phe) and poly(L-phenylalayl (3,4ethylenedioxythiophene-2'-yl)methylamide) (PEDOT-L-Phe) were synthesized by electrochemical polymerization, and their properties as an immobilization platform for biosensor application were explored. After covalent immobilization of ascorbate oxidase (AO) on the polymeric matrixs, their applications for ascorbic acid (AA) biosensing were investigated in detail. Importantly, optimized biosensors showed very good linear relationships between [AA] and the steady-state current response (I) in the concentration range of $0.03 \mu \mathrm{M}$ to $7 \mathrm{mM}(\mathrm{y}=\mathbf{0 . 0 8 9 7 2} \mathrm{x}+\mathbf{0 . 5 7 8 5}, \mathrm{r}=\mathbf{0 . 9 9 5 4}$, (PEDOT-Boc-Phe)) and $0.07 \mu \mathrm{M}$ to $7 \mathrm{mM}(\mathrm{y}=0.0397 \mathrm{x}+$ $0.59381, r=0.9928$ (PEDOT-Phe)), respectively. Satisfactory results implied that the as-formed polymer films were realized the immobilization of biologically active species and could be candidates in the development of biosensing performance.
\end{abstract}

Keywords-chiral poly(3,4-ethylenedioxythiophene); electropolymerization; amperometric biosensor; ascorbic acid

\section{INTRODUCTION}

Chiral polymers have attracted much attention because of their chiroptical activity [1], asymmetric separation [2] and recognition properties [3], and various types of chiral structure. Based on these advantages, it is now desirable to cultivate novel chiral polymers to achieve multifunctional and high-performance requirements. Chiral poly $(3,4-$ ethylenedioxythiophene) (PEDOT) is a representative of chiral polymers, which has attracted considerably widespread interest due to its versatile fascinating properties such as high electrical conductivity, low band gap, good redox activity, thermal stability, and excellent transparency in the doped state [4]. To date, there are several methods to obtain chiral PEDOTs. One of the most effective ways to induce such chiral structures in polymers is to introduce chiral moieties into the polymer structures [5]. Therefore, a series of new-generation chiral PEDOTs have been designed and synthesized to explore their promising potential applications. Importantly, to the best of our knowledge, the study of using the property of chiral PEDOT for the detection of chiral drugs still remains blank.

Vitamin $\mathrm{C}$ or ascorbic acid (AA), a water-soluble vitamin, is the chiral enolic form of an $\alpha$-ketolactone, which is widely present in many biological systems and in multivitamin preparations. AA plays a key role in the metabolism of cholesterol by increasing its elimination and thereby assisting lowering blood cholesterol [6]. Its deficiency leads to the development of a well-known syndrome called scurvy. It is administered in the treatment of many disorders, including Alzheimer's disease, atherosclerosis, cancer, and infertility as well as some clinical manifestations of HIV infections [7, 8]. There are various methods available for determination of AA such as colorimetry [9], high performance liquid chromatography [10], spectrophotometric methods [11], and sequential injection spectrophotometry [12]. However, the operation of colorimetry and high performance liquid chromatography methods is more complex, and the cost is relatively high. Spectrophotometric and chromatographic methods usually require sample pretreatment (e.g., extraction, complex formation) that is laborious and time-consuming. To overcome these defects, electrochemical methods are used extensively for the elegant and sensitive properties such as selectivity, simplicity, and reproducibility of this approach.

In this work, two novel PEDOT derivatives, poly ( $\mathrm{N}$ (tert-butoxycarbonyl)-L-phenylalayl $(3,4-$ ethylenedioxythiophene-2'-yl)methylamide) (PEDOT-BocL-Phe) and poly(L-phenylalayl (3,4-ethylenedioxythiophene2'-yl)methylamide) (PEDOT-L-Phe), were electropolymerized (Scheme 1). A simple and fast procedure was used for the fabrication of electrodes modified with chiral PEDOT-Boc-L-Phe (PEDOT-Boc-L-Phe/AO/GCE) and PEDOT-L-Phe (PEDOT-L-Phe/AO/GCE). The obtained electrodes for determination of AA by steady-state current response in phosphate buffer solution as the supporting electrolyte was described. Based on the effective electrocatalytic activity of the modified electrodes toward these species, a sensitive electrochemical sensor for determination of AA was established. Finally, the analytical 
performance of this sensor for determinations of AA in some commercial pharmaceutical samples was evaluated.

\section{EXPERIMENTAL}

\section{A. Materials}

$\mathrm{N}$-(3-Dimethylaminopropyl)-N'-ethylcarbodiimide hydrochloride (EDC HCl, 99\%), and $\mathrm{N}$-hydroxysuccinimide (NHS, 99\%) were purchased from Aladdin Chemistry Co. Ltd. Ascorbate oxidase (AO, $400 \mathrm{U} \mathrm{mg}^{-1}$ ) was purchased from J\&K. Phosphate-buffered solution (PBS, pH 7.0) was prepared from $0.1 M$ sodium dihydrogen phosphate dihydrate $\left(\mathrm{NaH}_{2} \mathrm{PO}_{4} \cdot 2 \mathrm{H}_{2} \mathrm{O}\right.$, Sinopharm chemical reagent Co., Ltd.) and $0.1 M$ disodium hydrogen phosphate dodecahydrate $\left(\mathrm{Na}_{2} \mathrm{HPO}_{4} \bullet 12 \mathrm{H}_{2} \mathrm{O}\right.$, Sinopharm chemical

\section{B. Fabrication of biosensor}

The glassy carbon electrode (GCE) electrode with a diameter of $3 \mathrm{~mm}$ served as the working electrode, a platinum wire with a diameter of $0.5 \mathrm{~mm}$ was used as an auxiliary electrode and a saturated calomel electrode (SCE) was used as the reference electrode. Then, the counter electrode and the GCE was ultrasonically cleaned in turn with deionized distilled water, ethanol, and deionized distilled water each for $5 \mathrm{~min}$, respectively. Finally, they were dried in air before the experiment. Three electrodes in the cell were placed $5 \mathrm{~mm}$ apart during electrochemical measurements. The PEDOT-Boc-L-Phe film was performed by cyclic voltammograms (CVs) from $-0.5 \mathrm{~V}$ to $1.5 \mathrm{~V}$ in $\mathrm{CH}_{2} \mathrm{Cl}_{2}$ and $0.1 \mathrm{M}$ terabutylammonium hexafluorophosphate system $\left(\mathrm{CH}_{2} \mathrm{Cl}_{2}-\mathrm{Bu}_{4} \mathrm{NPF}_{6}(0.1 \mathrm{M})\right)$ with a speed of $100 \mathrm{mV} \mathrm{s}$ 1 at room temperature. The PEDOT-L-Phe film was performed by $\mathrm{CVs}$ from $-0.2 \sim 0.8 \mathrm{~V}$ in $\mathrm{CH}_{2} \mathrm{Cl}_{2}-\mathrm{Bu}_{4} \mathrm{NPF}_{6}(0.1$ $M$ ) containing $3 \%$ TFA with a speed of $100 \mathrm{mV} \mathrm{s}^{-1}$ at room temperature. The obtained PEDOT-L-Phe modified GCE electrode was washed repeatedly with double-distilled deionized water to remove the electrolyte and monomer, and dried in air.

In bioactive platform preparation, EDC and NHS were used as cross linkers to obtain a covalent binding between amino groups of the polymer and carboxylic acid groups of the enzyme molecules [15-17]. The enzyme solution that contained $3 \mu \mathrm{L}$ (3.33 U) AO, $3 \mu \mathrm{L} 0.4 M$ EDC and $3 \mu \mathrm{L} 0.1$ $M$ NHS was dip-coated on the surface of the polymer coated electrode [18]. The modified electrodes were stored in a refrigerator $\left(4^{\circ} \mathrm{C}\right)$.

\section{RESUlts AND Discussion}

\section{Amperometric detection of $A A$}

The amperometric response of the PEDOT-LPhe/AO/GCE to successive adding AA was further evaluated under the optimized experimental conditions. Fig. 1 showed the typical current-time dynamic response of PEDOT-BocL-Phe/AO/GCE (A) and PEDOT-L-Phe/AO/GCE (B) towards AA. The concentration of $\mathrm{O}_{2}$ was kept constantly by air-saturating buffer solutions because $\mathrm{O}_{2}$ was a co-substrate reagent Co., Ltd.). Ascorbic acid (AA) was purchased from Bio Basic Inc. All chemicals were used without any further purification. EDOT-Boc-L-Phe and EDOT-L-Phe were prepared in accordance with Duan et al $[13,14]$.

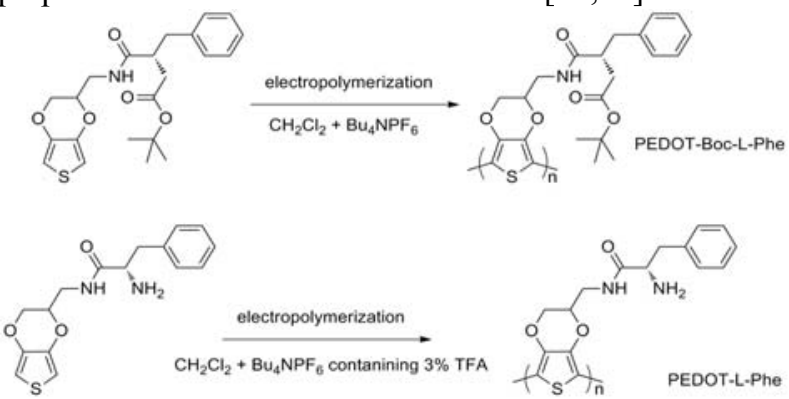

Scheme 1 Electropolymerization of EDOT-Boc-L-Phe and EDOT-L-Phe

of AO catalytic reaction. The steady-state current response increased with the increasing concentrations of AA revealing that AA could be detected successfully by the fabricated biosensor. The linear range of the steady-state current response (I) and the concentration of $\mathrm{AA}$ ([AA]) were defined at lower concentration.

The biosensor responses for varying AA concentrations were recorded under optimized conditions using $0.38 \mathrm{~V}$ as

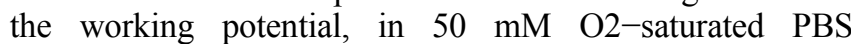
$(\mathrm{pH}=7.0)$ under stable stirring condition at room temperature. The results indicated that this biosensor exhibited an excellent current response for AA with short response time (within $10 \mathrm{~s}$ ). Good linear relationship was observed between [AA] and

$\mathrm{I}$ in the concentration range of $0.03 \mu \mathrm{M}$ to $7 \mathrm{mM}(\mathrm{y}=$ $0.08972 \mathrm{x}+0.5785, \mathrm{r}=0.9954$, (Fig. 1A)) and $0.07 \mu \mathrm{M}$ to $7 \mathrm{mM}(\mathrm{y}=0.0397 \mathrm{x}+0.59381, \mathrm{r}=0.9928$ (Fig. 1B)), respectively. The sensitivity (from the slope of the linear part in Fig. 1B) was $0.5785 \mu \mathrm{M}$ and $0.59381 \mu \mathrm{M}$, respectively. The detection limits were estimated to be $0.01 \mu \mathrm{M}$ and 0.023 $\mu \mathrm{M}$ (based on $\mathrm{S} / \mathrm{N}=3$ ) for $\mathrm{AA}$. In addition, the higher concentration of $\mathrm{AA}$ was not studied owing to the consumption of the dissolved $\mathrm{O}_{2}$ amounts in surrounding enzymes resulting in a gradual deviation from linearity according to our previous reports $[19,20]$. The performances of the fabricated sensor were compared with different AA sensors and the results indicated that this method was reasonable for detecting the unknown [AA] and could determine the content of AA in plants within the narrow limitations of $2 \times 10^{-3}$ to $2.5 \times 10^{-2} \mathrm{M}[21]$.

\section{CONCLUSION}

In this study, chiral PEDOT-Boc-L-Phe/AO/GCE and PEDOT-L-Phe/AO/GCE electrodes were prepared by electrochemical polymerization method and used as a novel sensing platform for electrochemical detection of AA. The GCE electrodes modified with PEDOT-Boc-L-Phe and PEDOT-L-Phe compositing with AO showed high electrocatalytic activity toward the determination of AA and exhibited good linear dependence and selectivity to AA concentration change. These results indicated that the new 
composite was scientifically interesting and had great potential for use in sensors. This can be a promising approach for detection of AA and may open new opportunities for facile, biocompatible, sensitive and robust chiral assays in biochemical applications.

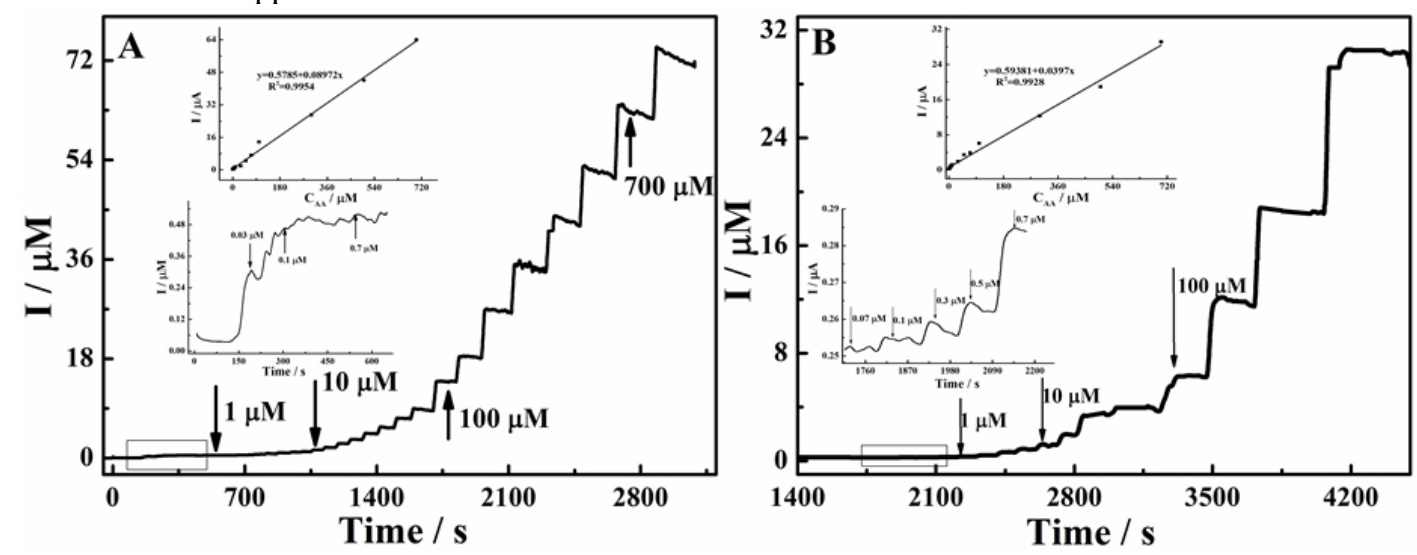

Figure 1. The constant potential amperometric response curves of AA at $0.38 \mathrm{~V}$ in oxygen saturated PBS under stable stirring condition at room temperature.

\section{ACKNOWLEDGMENT}

The authors would like to acknowledge the financial support of this work by the National Natural Science Foundation of China (51263010, 51303073, 51272096), Natural Science Foundation of Jiangxi Province (20114BAB203015, 20122BAB216011, 20142BAB206028) and the Science and Technology Landing Plan of Universities in Jiangxi province (KJLD14069).

\section{REFERENCES}

[1] J. M. Yu, T. Sakamoto, K. Watanabe, S. Furumi, N. Tamaoki, Y. Chen and T. Nakano, "Synthesis and efficient circularly polarized light emission of an optically active hyperbranched poly(fluorenevinylene) derivative," Chem. Commun., vol. 47, pp. 3799-3801, February 2011.

[2] K. Tamura, T. Miyabe, H. Iida and E. Yashima, "Separation of enantiomers on diastereomeric right- and left-handed helical poly(phenyl isocyanide)s bearing L-alanine pendants immobilized on silica gel by HPLC," Polym. Chem., vol. 2, pp. 91-98, August 2010.

[3] X. Y. Du, J. B. Liu, J. P. Deng and W. T. Yang, "Synthesis and chiral recognition of optically active hydrogels containing helical polymer chains," Polym. Chem., vol. 1, pp. 1030-1038, May 2010.

[4] Y. S. Jeong and K. Akagi, "Control of chirality and electrochromism in copolymer-Type chiral PEDOT derivatives by means of electrochemical oxidation and reduction," Macromolecules, vol. 44, pp. 2418-2426, March 2011.

[5] K. Kawabata and H. Goto, "Synthesis and optical properties of 1,1binaphthyl-thiophene alternating copolymers with main chain chirality," J. Mater. Chem., vol. 22, pp. 23514-23524, September 2012.

[6] N. Chauhan, J. Narang and C. S. Pundir, "Fabrication of multiwalled carbon nanotubes/polyaniline modified Au electrode for ascorbic acid determination," Analyst, vol. 136, pp. 1938-1945, March 2011.

[7] R. Aguilar, M. M. Dávila, M. P. Elizalde, J. Mattusch and R. Wennrich, "Capability of a carbon-polyvinylchloride composite electrode for the detection of dopamine, ascorbic acid, and uric acid," Electrochim. Acta, vol. 49, pp. 851-859, March 2004.
[8] B. Habibi, M. Jahanbakhshi and M. H. Pournaghi-Azar, "Differential pulse voltammetric simultaneous determination of acetaminophen and ascorbic acid using single-walled carbon nanotube-modified carbonceramic electrode," Anal. Biochem., vol. 411, pp. 167-175, April 2011.

[9] M. Polášek, P. Skála, L. Opletal and L. Jahodář, "Rapid automated assay of anti-oxidation/radical-scavenging activity of natural substances by sequential injection technique (SIA) using spectrophotometric detection," Anal. Bioanal. Chem., vol. 379, pp.754-758, July 2004.

[10] J. Lykkesfeldt, S. Loft and H. E. Poulsen, "Determination of ascorbic acid and dehydroascorbic acid in plasma by high-performance liquid chromatography with coulometric detection - are they reliable biomarkers of oxidative stress?," Anal. Biochem., vol. 229, pp. 329 335, August 1995.

[11]R. Sandulescu, S. Mirel and R. Oprean, "The development of spectrophotometric and electroanalytical methods for ascorbic acid and acetaminophen and their applications in the analysis of effervescent dosage forms," J. Pharm. Biomed. Anal., vol. 23, pp. 77-87, August 2000.

[12]K. Wimalasena and S. Dharmasena, "Continuous spectrophotometric assay for ascorbate oxidase based on a novel chromophoric substrate, 2-aminoascorbic acid," Anal. Biochem., vol. 210, pp. 58-62, April 1993.

[13]D. F. Hu, B. Y. Lu, X. M. Duan, J. K. Xu, L. Zhang, K. X. Zhang, S. M. Zhang, and S. J. Zhen, "Synthesis of novel chiral L-leucine grafted PEDOT derivatives with excellent electrochromic performances," RSC Adv., vol. 4, pp. 35597-35608, July 2014.

[14] L.Q. Dong, B.Y. Lu, X.M. Duan, J.K. Xu, D.F. Hu, K.X. Zhang, X.F. Zhu, H. Sun, S.L. Ming, Z.P. Wang, and S.J. Zhen, "Novel chiral PEDOTs for selective recognition of 3,4-dihydroxyphenylalanine enantiomers: synthesis and characterization," J. Polym. Sci. A Polym. Chem., in press. doi: 10.1002/pola.27695

[15]M. Kesik, F. E. Kanik, G. Hızalan, D. Kozanoglu, E. N. Esenturk, S. Timur and L. Toppare, "A functional immobilization matrix based on a conducting polymer and functionalized gold nanoparticles: Synthesis and its application as an amperometric glucose biosensor," Polymer, vol. 54, pp. 4463-4471, August 2013.

[16] S. Soylemez, F. E. Kanik, A. G. Nurioglu, H. Akpinar and L. Toppare, "A novel conducting copolymer: Investigation of its matrix properties 
for cholesterol biosensor applications," Sensors and Actuators B: Chemical., vol. 182, pp. 322-329, June 2013.

[17]F. Ekiz, F. Oğuzkaya, M. Akin, S. Timur, C. Tanyeli and L. Toppare, "Synthesis and application of poly-SNS-anchored carboxylic acid: a novel functional matrix for biomolecule conjugation," J. Mater. Chem., vol. 21, pp. 12337-12343, July 2011.

[18]N. C. Kekec, F. E. Kanik, Y. A. Udum, C. G. Hizliates, Y. Ergun and L. Toppare, "A novel conducting polymer based platform for ethanol sensing," Sensors and Actuators B: Chemical., vol. 193, pp. 306-314, March 2014.
[19]M. Liu, Y. P. Wen, D. Li, H. H. He, J. K. Xu, C. C. Liu, R. R. Yue, B. Y. Lu and G. D. Liu, "Electrochemical Immobilization of Ascorbate Oxidase in Poly(3,4-ethylenedioxythiophene)/Multiwalled Carbon Nanotubes Composite Films," J. Appl. Polym. Sci., vol. 122, pp. 11421151, May 2011.

[20]M. Liu, Y. P. Wen, D. Li, R. R. Yue, J. K. Xu and H. H. He, “A stable sandwich-type amperometric biosensor based on poly(3,4ethylenedioxythiophene)-single walled carbon nanotubes/ascorbate oxidase/nafion films for detection of L-ascorbic acid," Sensors and Actuators B: Chemical., vol. 159 pp. 277-285, November 2011.

[21]N. Smirnoff, "Ascorbic acid: metabolism and functions of a multifacetted molecule," Curr. Opin. Plant. Biol., vol. 3, pp. 229-235, 2000. 